\title{
Anxiety Disorders and Sensory Over-Responsivity in Children with Autism Spectrum Disorders: Is There a Causal Relationship?
}

\author{
Shulamite A. Green - Ayelet Ben-Sasson
}

Published online: 10 April 2010

(C) The Author(s) 2010. This article is published with open access at Springerlink.com

\begin{abstract}
Anxiety disorders and sensory over-responsivity (SOR) are common in children with autism spectrum disorders (ASD), and there is evidence for an association between these two conditions. Currently, it is unclear what causal mechanisms may exist between SOR and anxiety. We propose three possible theories to explain the association between anxiety and SOR: (a) SOR is caused by anxiety; (b) Anxiety is caused by SOR; or (c) SOR and anxiety are causally unrelated but are associated through a common risk factor or diagnostic overlap. In this paper, we examine support for each theory in the existing anxiety, autism, and neuroscience literature, and discuss how each theory informs choice of interventions and implications for future studies.
\end{abstract}

Keywords Autism spectrum disorders · Anxiety ·

Sensory over-responsivity · Hyperarousal .

Sensory defensiveness

Anxiety disorders are very common in children with autism spectrum disorders (ASD), and can increase the functional impairment of these children (Lainhart 1999; White et al. 2009). A number of studies have found rates of anxiety disorders and symptoms to be higher in children with ASD than in typically developing (TD) children (Gadow et al. 2004; Kim et al. 2000; Muris et al. 1998; Sukhodolsky et al.

S. A. Green $(\square)$

Department of Psychology, University of California,

1285 Franz Hall, Los Angeles, CA 90095, USA

e-mail: shulamite@ucla.edu

\section{A. Ben-Sasson}

Department of Occupational Therapy; Faculty of Social Welfare and Health Sciences, University of Haifa, Mount Carmel, Haifa, Israel
2008; Weisbrot et al. 2005) and children with intellectual disability (Brereton et al. 2006). Rate estimates of anxiety disorders range from 18 (Gadow et al. 2004) to 87\% (Muris et al. 1998) for children with ASD. Estimates of anxiety disorders in pre-adolescent TD children vary considerably but rates are thought to be between 3 and 24\% (CartwrightHatton et al. 2006). Sensory over-responsivity (SOR) is another common, and impairing feature found in more than half of children with ASD, and SOR has been linked to anxiety in children with ASD (Ben-Sasson et al. 2008; Liss et al. 2006; Pfeiffer et al. 2005). Reports of SOR rates of 56-70\% in children with ASD (Baranek et al. 2006; Ben-Sasson et al. 2007b) indicate elevated levels of SOR relative to rates of $10-17 \%$ in the general population (Ben-Sasson et al. 2009; Ben-Sasson et al. 2007b). Children with SOR often react negatively to noisy or visually complex environments, are bothered by tags or seams on their clothing, or may dislike being touched unexpectedly (Liss et al. 2006). While there is some evidence that anxiety and SOR are associated in children with ASD, it is unclear what causal mechanisms (if any) exist between them.

Although there are reports of the association of SOR and anxiety in individuals with other childhood and developmental disorders such as ADHD (Reynolds et al. 2009) we chose to focus on ASD for the following reasons: (a) There are elevated rates of both conditions in individuals with ASD so determining causality between SOR and anxiety in individuals with ASD has important implications for intervention choices in this population; (b) It is possible that factors unique to ASD put individuals with ASD at higher risk for both SOR and anxiety; and (c) ASD poses unique challenges to the differentiation of SOR from anxiety due to the lower functional communication and/or cognitive abilities of many individuals with ASD. These deficits may make it more difficult for individuals with 
ASD to report thought processes and sources of stress and for their parents to accurately read their facial expressions (Davis et al. 2008).

We propose three possible theories to explain the overlap between anxiety and SOR: (a) SOR is caused by (or is a symptom of) anxiety; (b) Anxiety is caused by (or is a symptom of) SOR; or (c) SOR and anxiety are not directly causally related, but are associated through a third variable such as a common risk factor or overlapping diagnostic criteria. We will examine support for each theory in the existing literature, drawing from research in the fields of anxiety, autism, and neuroscience. We will also discuss how each theory informs choice of interventions as well as implications for future studies.

\section{Anxiety as a Cause of Sensory Over-Responsivity: The Primary Anxiety Model}

Studies on hypervigilance and attention in individuals with anxiety disorders can inform our understanding of how anxiety might elicit SOR in children with ASD (see Fig. 1). Evidence indicates that anxiety disorders are characterized by hyperarousal (i.e., heightened baseline levels of autonomic arousal) which supports a state of hypervigilance (i.e., scanning the environment for threat-relevant stimuli, and preparation for potential threat). It is posited that, for anxious individuals, this hypervigilance is part of an overall difficulty in regulating negative affectivity, known as threat-based emotion regulation (Craske 2003). These individuals may have trouble shifting attention and activating or inhibiting behavior in such a way that decreases negative affectivity and arousal.

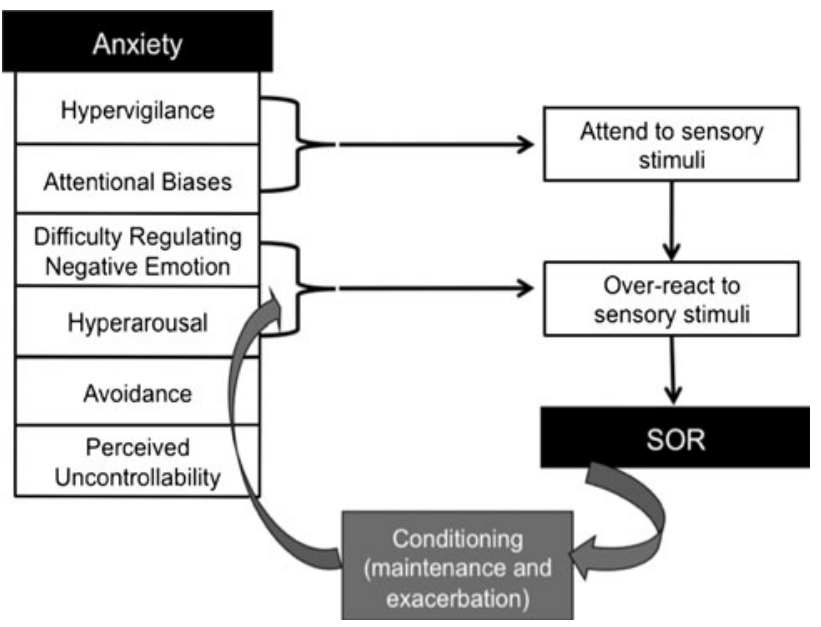

Fig. 1 Primary anxiety model portraying the theoretical pathway from symptoms of anxiety disorders to over-reaction to sensory stimuli. This pathway is then maintained and exacerbated through conditioning
Studies using paradigms that assess attentional bias support the theory that anxious individuals have higher rates of environmental scanning (i.e., hypervigilance), followed by a narrowing of attention once a threat-relevant stimulus is detected, and then relative difficulty disengaging from that stimulus (Craske et al. 2009; Mobini and Grant 2007). For example, in the dot probe task, individuals with anxiety disorders show slower response latencies to a dot on the opposite side of the screen from a threat-relevant image or word than a dot on the same side of the screen, relative to healthy controls. These findings have been replicated with children, suggesting that relative to healthy controls, children with anxiety disorders show a stronger bias in selectively attending to threat-relevant stimuli, and difficulty disengaging from these stimuli even when they have been asked to attend to other information (Mobini and Grant 2007).

This type of anxious hypervigilance and poor regulation of negative arousal through attentional bias could contribute to SOR. If children are hyperaroused and scanning the environment, looking for threat, they are more likely to notice and react to environmental sensory stimuli. A threatbased style of emotion regulation would exacerbate this reactivity, as they would be more likely to attribute threat to these stimuli, and have difficulty shifting their attention and inhibiting negative affect. A study by Liss et al. (2006) lends some support to the relevance of this model for children with ASD. Liss and colleagues showed that parent ratings of SOR, excellent memory, and overfocused and overselective attention cluster together in a subgroup of children with ASD. The authors suggested that these individuals overreact to aversive sensory stimuli because they are more likely to attend to them and have difficulty disengaging from them. Thus, this study supports the idea that there is a subgroup of children with ASD who are particularly prone to SOR, and that these children show some symptoms similar to individuals with anxiety disorders, including difficulty with rapid disengagement, and selective attention.

Experimental manipulation of anxiety and sensory sensitivity in animal research lends further support to the theory that anxiety may lead to SOR by altering an individual's ability to regulate his or her reactions to sensory input. In a study by Lepicard et al. (2003) inbred strains of mice that were genetically modified to be anxiety prone showed poorer balance and postural control than non-anxious mice. This may be an indicator of poor modulation of vestibularproprioceptive input, which is often observed in children with SOR (Miller et al. 2007). Administration of an anxiolytic pharmacological compound to the anxious mice and anxiogenic compounds to the normal mice reversed the group differences so that the originally normal mice had poorer balance and postural control than the originally anxious mice. This causal effect can be found in other 
sensory modalities as well. For example, Plappert and Pilz (2002) found that mice that were genetically anxiety prone showed a stronger auditory startle reaction to sounds than non-anxious mice. However, such results must be interpreted with caution because the auditory startle reaction is also a symptom of anxiety. More studies are necessary to examine the response of genetically-anxious animals to non-startling sensory stimuli in order to avoid confounding the sensory response with the anxiety response.

Once over-reactivity has been established through hyperarousal, hypervigilance, and threat-based emotion regulation, it may be maintained or exacerbated by classical aversive conditioning, in which an innately aversive event (such as a loud noise) is associated with a previously neutral stimulus (such as an electronic toy). For example, if a child with ASD is constantly scanning the environment for sensory-related threat and preferentially attends to aversive, unexpected noises, he or she is more likely to overreact to these noises. The sensory overreaction feels unpleasant, and the child associates this unpleasant feeling with the aversive noise. This pairing of a conditioned stimulus (the loud noise) with a physiological reaction (e.g., fight-or-flight response) is known as interoceptive conditioning and is present in many different anxiety disorders (Razran 1961). Symptoms associated with anxiety disorders including trait anxiety, physiological arousal, and the perceived uncontrollability of an aversive event all contribute to stronger conditioning (Craske 2003), thus increasing the likelihood that a child with a preexisting anxiety disorder might associate a physiological reaction to a sensory stimulus. Once conditioning occurs, the child is more likely to scan the environment for potential aversive stimuli (e.g., balloons, radios, telephones), more likely to selectively attend to those objects, so more likely to notice a sound and react negatively to it when it does occur. Furthermore, children with anxiety disorders tend to regulate their negative affectivity through avoidance of stimuli that elicit fear (Craske 2003). Avoidance further decreases the possibility that the child will learn to regulate his or her response to the stimuli in an adaptive way. In this way, conditioning serves to maintain or exacerbate the connection between anxious hypervigilance and SOR. Thus, SOR could be a result of hypervigilance, difficulty regulating reactions to aversive sensory stimuli, and then, through conditioning and avoidance, maintaining and strengthening the association of over-reactivity with those stimuli.

Model Limitations

Hyperarousal is Not Characteristic of All Individuals with $A S D$

In a review of physiological studies in children with ASD, Rogers and Ozonoff (2005) found no evidence for general hyperarousal in children with ASD. Their conclusion might seem to contradict a model of hyperarousal and hypervigilance causing SOR. However, the physiological studies reviewed included very small samples of children with ASD and were not specific to children with either anxiety or sensory dysregulation. There may be a subset of children with ASD who also have anxiety and/or SOR, for whom the hypervigilance theory is relevant.

Anxiety in Very Young and Low-Functioning Children with $A S D$

It is also important to ask whether, theoretically, a preverbal child with low cognitive ability can be anxious, as some theories of anxiety relate anxiety to conscious cognitive processing and anticipation of future events (e.g., Beck and Clark 1997). Children who are unable to process their reactions to aversive events might feel fear in the moment but lack the cognitive ability to anticipate that fear in the future. However, Mogg and Bradley (1998) describe anxiety as a preattentive bias towards threat, and theorize that the automatic encoding of threat without elaborative processing leads to anxiety. According to Mogg and Bradley's model, conscious cognitive awareness is not necessary for developing anxiety. Research on co-morbid anxiety across the autism spectrum supports this model. While anxiety is more common in children with higher IQs, anxiety disorders may be present in as many as $40 \%$ of children with ASD who have IQs under 70 (Sukhodolsky et al. 2008).

If anxiety causes SOR, then SOR in toddlers with ASD may be a marker of an underlying anxiety disorder. Very few toddlers (around 5-6\%) with ASD are identified as having high rates of anxiety (Ben-Sasson et al. 2008) but it is unclear whether that is due to actual low rates of anxiety or difficulty in identifying anxiety at this early age. Young children with ASD are more likely to have lower verbal skills and functional communicative ability than TD children, and may lack the symbolic ability to connect the source of their fear to their reaction. Some of these children also have a reduced range of facial affect and ability to express emotion appropriately (Yirmiya et al. 1989). Consequently, parents and therapists may recognize a child's over-responsiveness to sensory stimuli but may not be able to identify anxiety as the cause of the overresponsivity (Fox and Polak 2004). Studies of physiological hyperarousal, attention, and response to threat-relevant stimuli in children with ASD and SOR could help answer this question, since most studies of young children with ASD currently rely on parent report of both SOR and affective symptoms. 
Sensory Over-Responsivity as a Cause of Anxiety: The Primary SOR Model

SOR as a causal factor for anxiety can be conceptualized in terms of fear and conditioning (see Fig. 2). Unpleasant sensory stimuli (the unconditioned stimuli, such as an aversive noise) are associated with certain objects or situations, such as a balloon or an alarm clock. Consequently, these objects become conditioned stimuli, capable of eliciting a conditional response, such as fear or anxiety. Thus, the balloon may later elicit an anxious response without the presence of an aversive noise. Specific phobia is one of the most common anxiety disorders seen in children with ASD (Gadow et al. 2004; Muris et al. 1998; Sukhodolsky et al. 2008), which is consistent with this fear and conditioning model, as it is easy to imagine how children with SOR might develop phobic responses to stimuli associated with aversive sensory experiences such as balloons, elevators, scratchy clothing, etc. However, conditioning is also a mechanism through which SOR might lead to more generalized anxiety. For example, if the unconditioned stimulus does not always signal the conditioned stimulus (i.e., aversive noises can occur unexpectedly without being predicted by one particular object), the fear is more likely to generalize through what is called context conditioning (Grillon 2008). Through context conditioning, the conditional fear response can shift from being triggered by a specific object to a location or context in which the aversive stimulus has occurred, so the child might refuse to go to a birthday party or into a bedroom because they anticipate there might be aversive sounds at these locations. Animals who have experienced context conditioning become behaviorally avoidant (Bouton and King 1983), and likewise children with SOR often become avoidant of generalized locations such as restaurants, grocery stores, or parties in addition to specific objects (Schoen et al. 2008). The more generalized the avoidance, the more impaired the child is likely to be.

A number of factors have been found to contribute to the "conditionability" of a particular situation, including the intensity of the unconditional response and uncontrollability of the conditioned stimuli (Craske 2003). As children with SOR by definition have an intense unconditional response to sensory stimuli, and as these stimuli are often elicited through factors completely outside the children's control (Reynolds and Lane 2008), one can see how these children are likely at high risk for fear conditioning. Furthermore, the cognitive and language deficits often associated with ASD may make it more difficult for children to identify relationships of predictability and/or exert control over a stressful situation (e.g. by expressing fear in a way that will cause others to reduce the threat-relevant stimuli). The frequency, uncontrollability, and unpredictability of the conditioned stimuli may then cause the child to become generally hypervigilant to potentially threatening sensory stimuli, and to maintain physiological arousal, resulting in a state of generalized anxiety and worry.

It is possible that attentional bias also plays a role in how SOR contributes to anxiety. SOR may cause attentional bias towards the perceived threatening stimuli, and that attentional bias can cause negative mood states in response to stress (Craske 2003). In particular, MacLeod et al. (2002) demonstrated that training individuals to have an attentional bias towards threat-relevant words subsequently increased their negative response to stress. These results do not specifically address attentional bias as a causal factor for anxiety disorders, but do suggest that attentional bias could contribute to the development of anxiety.

\section{Model Limitation: Fear vs. Anxiety}

SOR is, almost by definition, a fearful response, but fear alone does not imply an anxiety disorder. Grillon (2008) differentiates between fear and anxiety: Fear is "a response to an impending identifiable danger...a surge of physiological arousal, an alarm reaction resulting in reflexive action" whereas anxiety is "a state of chronic apprehension about future harm, characterized by tension, worry, negative affect, and a feeling of insecurity...elicited by

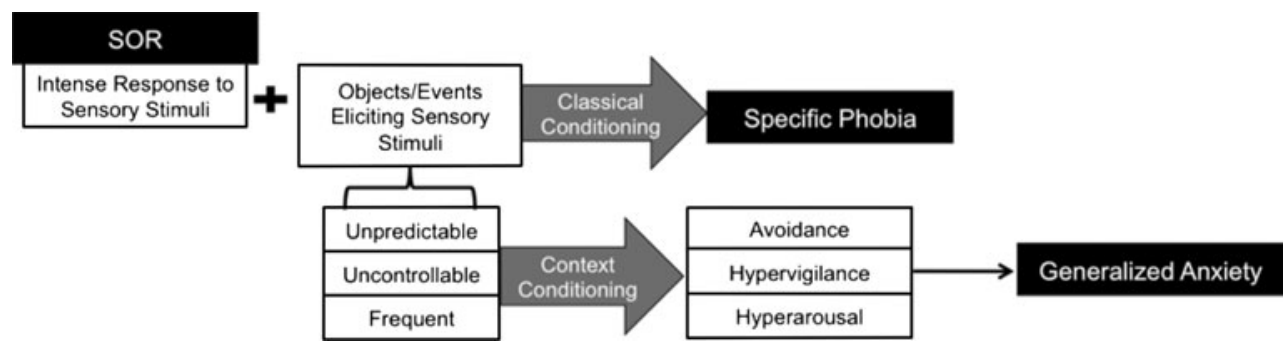

Fig. 2 Primary SOR model portraying the theoretical pathway from symptoms of sensory over-responsivity to anxiety disorders. SOR may lead to specific phobia through classical conditioning.
Additionally, due to the unpredictability, uncontrollability, and frequency of sensory stimuli in the environment, it may lead to generalized anxiety through context conditioning 
unpredictability and by the perception of potential, unseen, or symbolic threats" (p. 422).

Most anxiety disorders are a combination of a specific conditioned fear and anticipation of the feared stimulus. Because context conditioning is associated with unpredictability and hippocampus activation (Grillon 2008), it is more characteristic of anxiety than fear. Context conditioning has been studied using virtual reality environments (Grillon) and these studies could be replicated in children with ASD and SOR to study whether they anticipate threat in an unpredictable environment or do not act fearful until a particular stimulus is present.

\section{Non-causal Explanations for the Association Between SOR and Anxiety}

We must consider the possibility that SOR and anxiety are not directly causally related, but are associated through a third variable such as a common risk factor (e.g., amygdala abnormalities) or through overlapping diagnostic criteria (see Fig. 3).

\section{Connection Through Amygdala Abnormalities}

It is possible that amygdala abnormalities contribute to both anxiety and SOR. The amygdala has been implicated in anxiety disorders, mainly due to its central role in fear conditioning (e.g., Davis 1992; Garakani et al. 2006; Rauch et al. 2003). There is some support for the theory that amygdala abnormalities also contribute to SOR. The amygdala plays a central role in the detection and response to threat (Zald 2003), and research associating the amygdala specifically with fearful responses to sensory stimuli supports the primary SOR model. Zald (2003) reviewed studies showing that the amygdala receives sensory input from auditory and visual sensory areas of the cortex, and that the perceived unpleasantness of a stimulus is correlated with the amount of amygdala activation. The

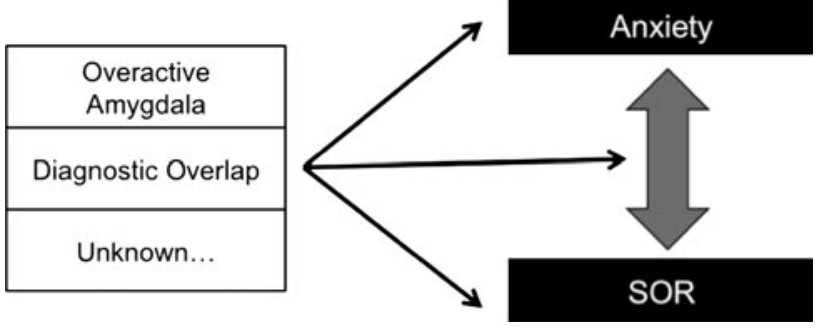

Fig. 3 Third model demonstrating how a third variable might explain the association between SOR and anxiety. This relationship could be caused by a general risk factor to both conditions such as an overactive amygdala, or be due to diagnostic overlap amygdala projects to cholinergic and noradrenergic cells, which can focus attention on the aversive stimulus, and to cortical sensory regions which may trigger an enhanced sensory response to emotionally-arousing or conditioned stimuli. The amount of enhancement in the sensory response is correlated with the amount of amygdala activation, which suggests that children with SOR may have over-active amygdalas (Zald 2003).

Additionally, the evidence that children with ASD and SOR tend to have overfocused attention and exceptional memory (Liss et al. 2006) further supports the role of the amygdala in SOR. The amygdala projects to the hippocampus (Stein et al. 2007), so is involved in encoding and strengthening memories of aversive events. An overactive amygdala may partially account for their good memory skills. Findings of amygdala abnormalities in some children with autism are consistent with the high rates of anxiety disorders and SOR in this population. There is evidence that children with ASD have increased amygdala volumes compared to typically developing children, and that amygdala size is positively correlated with anxiety and severity of social-communication symptoms in these children (Amaral et al. 2008; Juranek et al. 2006). Juranek et al. found a greater amygdala volume in children with ASD who were rated by their parents as having high symptoms of anxiety even after controlling for age, brain size, and severity of ASD symptoms. A larger amygdala might account for an overactive fear response in these children, and put them at risk for both anxiety disorders and SOR. Markram et al. (2008) examined the role of the amygdala in rats with ASD-like symptoms including repetitive behavior and reduced play and social behavior. The rats were also severely impaired in their ability to extinguish a conditioned fearful response to an aversive stimulus, and were more likely than normal rats to generalize fear responses to new contexts. The rats had twice the amygdala activation in response to electrical stimulation as normal rats as well as increased long-term potentiation (the mechanism through which memories are consolidated). The results of this study suggest that amygdala over-activation may lead to over-encoding and over-generalization of the fear response. These findings are consistent with the idea that children with autism may be more likely to have overactive amygdalas which may contribute to their development of SOR and anxiety disorders. According to this model, amygdala abnormalities independently contribute to SOR and anxiety, but the two conditions may still exacerbate each other as described in the Primary Anxiety and Primary SOR models. It may be that amygdala abnormalities in ASD can explain the higher rates of SOR and anxiety disorders in this population, but more research is necessary before coming to any conclusions on this topic. 


\section{Connection Through Diagnostic Overlap}

It is also possible that SOR and anxiety are unrelated, but appear to be correlated due to diagnostic overlap. The two conditions can be difficult to distinguish, as they both involve misperception of threat (e.g., Schoen et al. 2008) and overlap in their physiological (e.g., increased heart rate and skin conductance response) and behavioral (e.g., fight-or-flight response, emotional dysregulation, avoidance) symptoms. For those reasons, similar measures are often used to assess both problems. For example, cortisol levels have been used to measure stress levels in studies of both SOR and anxiety (e.g., McIntosh et al. 1999; Reynolds et al. 2009; Romancyzk and Gillis 2006). These physiological indicators support the fact that the child with SOR and/or anxiety is in a state of stress, but they do not provide means for differential diagnosis.

Behavioral overlap is also evident in parent-report measures. Scales of SOR and anxiety disorders include behavioral items that may indicate either SOR or anxiety, such as avoidance of mess and startle response to sound. This item overlap compromises attempts to study the association between these conditions using parentreport measures. Nevertheless, studies that document an association between anxiety and SOR in children with ASD often rely on parent report questionnaires that overlap in this way. Ben-Sasson et al. (2007a) investigated this problem by studying how occupational therapists differed from psychologists in their judgment of symptoms as representative of SOR versus anxiety in toddlers. The authors found that occupational therapists tended to rate items as representing SOR while psychologists tended to rate the same items as representing anxiety disorders. There were six items from anxiety or SOR scales that at least $80 \%$ of psychologists and occupational therapists rated as indicators of both conditions. In addition, in response to a vignette case study representing SOR, all occupational therapists diagnosed SOR while $26 \%$ of psychologists diagnosed an anxiety disorder, whereas in response to a vignette case study representing general anxiety 50\% of occupational therapists diagnosed SOR and $92 \%$ of psychologists diagnosed an anxiety disorder.

Thus, both professional background and item overlap may contribute to diagnostic overlap. Investigators studying the association between SOR and anxiety in children with ASD need to be particularly careful in how they identify each condition and, if the child enters the study with a diagnosis, what type of provider gave the diagnosis. There is need for more studies that integrate paradigms from occupational therapy and psychology to create more consistent diagnoses between the two fields.

\section{Implications for Intervention}

The choice of the most appropriate intervention for children with ASD who show anxiety and SOR symptoms depends on which causal pathway is supported. The two conditions are currently treated very differently, and as if they are entirely separate disorders. The most common interventions for SOR include Sensory Integration therapy (SI), the Sensory Diet, sensory stimulation (SS) techniques, and Auditory Integration Therapy (AIT), but evidence for the efficacy of these interventions is quite mixed and primarily based on case-study designs (see Baranek 2002 for a review). Sensory-based interventions aim to increase participation by reducing negative responses to sensations and/or improving sensory modulation. SI uses somatosensory and vestibular activities to improve children's ability to modulate, organize and integrate information from the environment. It is still a controversial therapy, and while some studies show that it can improve children's play and social-communication skills, there is little evidence that it specifically reduces SOR (Ayres and Tickle 1980; Case-Smith and Bryan 1999; Linderman and Stewart 1999). The Sensory Diet is a program of scheduled sensory activities that meet the child's sensory needs and involves frequent and systematic application of somatosensory stimulation. Although commonly used, there are no studies of its sensory or emotional outcome for children with ASD (Baranek). Likewise, Auditory Integration Training (AIT), which uses carefully modulated sounds to enhance auditory perception, has not been shown to be consistently efficacious in reducing auditory sensitivity (Baranek). The evidence for SS techniques is more promising. SS is based on the principle that deep pressure can influence the nervous system in a way that helps the child regulate his or her arousal level. For example, weighted vests which provide continuous pressure (Ferterl-Daly et al. 2001) or touch therapy (e.g., massage; Baranek; Field et al. 1997; Silva et al. 2009) have been shown to improve young children with ASD's responsiveness to sensory input, attention, stereotyped movements, and social-communication symptoms. The only ASD sensory intervention study that used anxiety as an outcome measure was conducted by Edelson et al. (1999). In this study the "hug machine" developed by Temple Grandin, which provides lateral body pressure, has been found to decrease tension and hyperactivity as well as anxiety in children with ASD, especially in children with higher initial levels of arousal or anxiety. This is consistent with work outside the ASD literature showing a decrease in secondary anxiety symptoms in adults with SOR following a sensory-based intervention (Pfeiffer and Kinnealey 2006). Therefore, it appears that certain sensory-based interventions can be successful in reducing anxiety in children with ASD. More rigorous evidence (e.g., from a 
randomized controlled treatment design) that shows the reduction of anxiety as an outcome of sensory-based interventions for children with ASD would support the Primary SOR model.

Recently, a few studies have shown Cognitive Behavioral Therapy (CBT) to be efficacious in treating anxiety in children with ASD (e.g., Chalfant et al. 2007; Reaven and Hepburn 2003; Sofronoff et al. 2005; Sze and Wood 2007; Wood et al. 2009). The CBT used in these studies was based on CBT for typically developing children, and included psychoeducation, cognitive restructuring, selftalk, relaxation, and exposure to feared stimuli. Chalfant et al. and Sofronoff et al. adapted CBT for children with ASD through methods such as simplifying the cognitive aspects and emphasizing concrete aspects such as relaxation and exposure. Moreover, Wood et al. enhanced a manualized CBT intervention for children with ASD by addressing poor social and adaptive skills in addition to the core anxiety problems. In all of these studies, children in the intervention groups had a significant reduction in anxiety, as compared to wait-list controls.

Psychopharmacological treatments, and specifically SSRIs, have been shown to decrease anxiety in children with ASD (White et al. 2009). SSRIs used to treat anxiety in children may also reduce amygdala volume (Szeszko et al. 2004). It seems possible, therefore, that if an overactive amygdala is implicated in SOR, SSRIs might also reduce SOR by reducing amygdala volume and/or activity.

To our knowledge no one has yet investigated the effect of anxiety interventions such as CBT or exposure therapy on SOR in children with ASD and anxiety. If anxiety is a causal factor for SOR or if SOR is a marker for unrecognized anxiety in young children, then sensory interventions need to take into account the effects of anxiety, and perhaps incorporate cognitive and behavioral strategies from evidence-based anxiety interventions. Likewise, if SOR is a causal factor for anxiety, then cognitive-behavioral programs for children with ASD and anxiety need to assess and treat sensory problems as well. Either way, children might benefit from an integrated intervention approach. For example, exposure therapy might be beneficial to children with primary SOR in addition to those with primary anxiety. Future studies are needed to examine whether young children with SOR who undergo exposure therapy are less likely to develop anxiety later on, which would support the primary SOR model. Exposure therapy might influence SOR through the following mechanism: exposure to fearful objects might provide an opportunity for children to become more aware of the contingencies for when aversive events occur and less likely to experience generalized context conditioning and behavioral avoidance. Exposure therapy could be combined with sensory stimulation intervention, such as body pressure, which would help decrease arousal (Baranek 2002) and make exposure less aversive. Regardless of which causal pathway is supported, pairing sensory-based interventions with CBT or exposure therapy might markedly increase a child's tolerance for previously aversive sensory stimuli and overwhelming environments.

The discipline of the professional evaluating the child is not only likely to impact the child's diagnosis but also the type of intervention the child is referred to. This problem was demonstrated by Ben-Sasson et al. (2007a) who found that occupational therapists were more likely to recommend a sensory-based intervention for case studies designed to represent a child with SOR or a child with generalized anxiety disorder whereas psychologists were more likely to recommend family therapy for either case. Professionals from all disciplines would thus benefit from a better understanding of the two conditions through education and training about assessments and interventions available across disciplines. When evaluating a child who presents with symptoms of SOR or anxiety, the professional should consider both for differential diagnosis and accurate referral.

An interdisciplinary framework of care is important but not sufficient given the overlap between assessments for SOR and anxiety. There is a need to develop distinct indicators to better differentiate the two. One way in which the two may be distinguished behaviorally is that SOR should be generalizable to multiple stimuli that elicit the unpleasant sensory response (e.g., several stimuli that create an aversive noise; see Miller et al. 2007) while specific phobia would be to one specific stimulus or situation (e.g. Ollendick et al. 2002). Furthermore, if a child has a general anxiety disorder, by definition he or she should be generally anxious, not only to specific sensory stimuli (e.g., Masi et al. 2004). Developing parent report and observation measures specific to ASD (Davis et al. 2008) that make such distinctions is needed to analyze sources of stress in the frequent case that children are too young or lack the verbal skills to report their own symptoms. Similarly, identifying distinct physiological indicators for clinical use would be advantageous for evaluating SOR and anxiety in ASD, independent of social and cognitive level. These methods can assist professionals across disciplines in considering the possibility of either condition as the primary cause of fear responses in ASD.

\section{Summary and Conclusions}

To summarize, we have proposed two theoretical, causal models that might explain the association between anxiety and SOR. While these two conditions are related in other populations, we chose to focus on children with ASD, 
because both conditions are common in and impairing to children with ASD and may be particularly challenging to differentiate in these children. In the Primary Anxiety model, anxiety contributes to SOR as generalized hyperarousal and hypervigilance focuses attention on a specific type of sensory stimulus. In this case, children who are hypervigilant and continuously scanning their environment are more likely to notice and react to aversive sensory stimuli in the environment. The threat-based emotion regulation associated with anxiety also makes it more difficult for children to regulate their emotional and physiological reactions to stimuli. The reaction may then worsen through both classical and interoceptive conditioning. In the Primary SOR model, SOR contributes to anxiety as a specific over-reaction generalizes to an environment or situation through context conditioning. The strength of the unconditional sensory reaction and the uncontrollability of events that elicit sensory stimuli contribute to stronger conditioning. We also addressed the possibility that a common risk factor such as amygdala abnormalities may contribute independently to each condition. The amygdala has long been known to play a role in fear and anxiety, and may also be related to SOR through overestimation of the threat value of a sensory stimulus which triggers an enhanced response to that stimulus.

In reality, these models oversimplify the development of these two conditions in children with ASD, as multiple genetic and environmental factors probably interact to contribute to the etiology and course of both anxiety and SOR. However, the models provide a theoretical basis from which to develop studies that can help us better understand the association between anxiety and SOR. Potentially informative studies include:

1. Studies that establish distinct parent-report and physiological measures of SOR and anxiety. Such studies are necessary to examine which stimuli are most aversive to children with ASD and SOR, with and without co-morbid anxiety disorder. For example, children who are over-responsive to sound should show over-responsivity to a variety of auditory stimuli whether or not they have co-morbid anxiety. On the other hand, children with ASD who are anxious but who do not have co-morbid SOR should show over-responsivity to a variety of startling stimuli, regardless of the modality of the stimulus.

2. Longitudinal, prospective studies beginning in infancy that involve assessing children for both SOR and anxiety at multiple time points, using distinct measures. Currently, SOR can be identified in infants later diagnosed with ASD (Zwaigenbaum et al. 2005), which is much earlier than anxiety is usually diagnosed. Longitudinal studies that find SOR consistently preceding anxiety in children with ASD would support the Primary SOR model, whereas studies that find children who do not have SOR at a young age develop it after being diagnosed with anxiety would promote the Primary Anxiety model. While such studies can only suggest causality and not prove it, they would have extremely valuable implications for intervention. For example, a finding that SOR predicts anxiety in children with SOR would suggest preventative anxiety interventions for these children. Furthermore, it is possible that there is a transactional relationship between anxiety and SOR, so longitudinal studies should examine whether the two covary over time and whether children with both conditions have more severe symptoms of anxiety or SOR than children with only one of the two.

3. Physiological studies that include adequate sample sizes and control groups (as suggested by Rogers and Ozonoff 2005), use sensory stimuli that are aversive to children with ASD, and examine subgroups of children with ASD, particularly those with anxiety symptoms. Such studies can help determine whether over-responsivity is characteristic of a specific subgroup of children with ASD. Additionally, imaging studies should examine possible amygdala over-reactivity in children with ASD and SOR with and without anxiety, as well as other brain areas which may be involved in SOR, such as the hippocampus.

4. Intervention studies that examine whether (a) interventions for sensory dysregulation decrease symptoms of anxiety; (b) interventions for anxiety decrease symptoms of SOR; and (c) combined interventions have greater effectiveness in reducing either condition in children with ASD.

5. Animal studies that use experimental conditions to test causal effects. It is difficult to determine causality in human studies, as we cannot manipulate SOR or anxiety in children. However, such studies are more feasible with animal populations, in which we can induce symptoms of SOR or anxiety to see if, and under what conditions, they develop symptoms of the other condition.

Studies such as those listed above would be valuable in furthering our understanding of the association between SOR and anxiety, in all populations, but especially in children with ASD. SOR and anxiety are particularly common and impairing in this population, so the hope is that such research would lead to an improved understanding of the etiology of both conditions, as well as more effective interventions and improved functioning for children with ASD. 
Acknowledgments This manuscript required the integration of multiple disciplines, and thus the authors are extremely grateful to have had input from individuals in the fields of occupational therapy, neuroscience, anxiety, and autism, and in particular Michelle Craske, Ph.D., Bram Vervliet, Ph.D., and David Shirinyan, Ph.D. The author Shulamite A. Green was supported in part by the Eunice Kennedy Shriver National Institute of Child Health and Human Development, Grant number: 34879-1459 (Drs. Keith Crnic, Bruce Baker, and Jan Blacher PIs). The author Ayelet Ben-Sasson was supported by a European International Reintegration Grant (\# 203715).

Open Access This article is distributed under the terms of the Creative Commons Attribution Noncommercial License which permits any noncommercial use, distribution, and reproduction in any medium, provided the original author(s) and source are credited.

\section{References}

Amaral, D. G., Schumann, C. M., \& Nordahl, C. W. (2008). Neuroanatomy of autism. Trends in Neuroscience, 31(3), 137-145.

Ayres, A. J., \& Tickle, L. S. (1980). Hyper-responsiveness to touch and vestibular stimuli as a predictor of positive response to sensory integration procedures by autistic children. American Journal of Occupational Therapy, 34(6), 375-381.

Baranek, G. T. (2002). Efficacy of sensory and motor interventions for children with autism. Journal of Autism and Developmental Disorders, 32(5), 397-422.

Baranek, G. T., David, F. J., Poe, M. D., Stone, W. L., \& Watson, L. R. (2006). Sensory experience questionnaire: Discriminating sensory features in young children with autism, developmental delays, and typical development. Journal of Child Psychology and Psychiatry, 47(6), 591-601.

Beck, A. T., \& Clark, D. A. (1997). An information processing model of anxiety: Automatic and strategic processes. Behavior Research and Therapy, 35(1), 49-58.

Ben-Sasson, A., Carter, A. S., \& Briggs-Gowan, M. (2009). Prevalence and correlates of sensory over-responsivity from infancy to elementary school. Journal of Abnormal Child Psychology, 37(5), 705-716.

Ben-Sasson, A., Cermak, S. A., Orsmond, G. I., Carter, A. S., \& Fogg, L. (2007a). Can we differentiate sensory over-responsivity from anxiety symptoms in toddlers? Perspectives of occupational therapists and psychologists. Infant Mental Health Journal, 28, 536-558.

Ben-Sasson, A., Cermak, S. A., Orsmond, G. I., Carter, A. S., Kadlec, M. B., \& Dunn, W. (2007b). Extreme sensory modulation behaviors in toddlers with autism. American Journal of Occupational Therapy, 61(5), 584-592.

Ben-Sasson, A., Cermak, S. A., Orsmond, G. I., Tager-Flusberg, H., Kadlec, M. B., \& Carter, A. S. (2008). Sensory clusters of toddlers with autism spectrum disorders: Differences in affective symptoms. Journal of Child Psychology and Psychiatry, 49(8), 817-825.

Bouton, M. E., \& King, D. A. (1983). Contextual control of the extinction of conditioned fear: Tests for the associative value of the context. Journal of Experimental Psychology: Animal Behavior Processes, 9, 248-265.

Brereton, A. V., Tonge, B. J., \& Einfeld, S. L. (2006). Psychopathology in children and adolescents with autism compared to young people with intellectual disability. Journal of Autism and Developmental Disorders, 36, 863-870.

Cartwright-Hatton, S., McNicol, K., \& Doubleday, E. (2006). Anxiety in a neglected population: prevalence of anxiety disorders in preadolescent children. Clinical Psychology Review, 26(7), 817-833.

Case-Smith, J., \& Bryan, T. (1999). The effects of occupational therapy with sensory integration emphasis on preschool-age children with autism. American Journal of Occupational Therapy, 53, 489-497.

Chalfant, A. M., Rapee, R., \& Carroll, L. (2007). Treating anxiety disorders in children with high functioning autism spectrum disorders: A controlled trial. Journal of Autism and Developmental Disorders, 37, 1842-1857.

Craske, M. G. (2003). Origins of phobias and anxiety disorders: Why more women than men?. Oxford: Elsevier.

Craske, M. G., Rauch, S. L., Ursano, R., Prenoveau, J., Pine, D. S., \& Zinbarg, R. E. (2009). What is an anxiety disorder? Depression and Anxiety, 26, 1066-1085.

Davis, M. (1992). The role of the amygdala in fear and anxiety. Annual Review of Neuroscience, 15, 353-375.

Davis, E., Saeed, S. A., \& Antonacci, D. J. (2008). Anxiety disorders in persons with developmental disabilities: Empirically informed diagnosis and treatment. Psychiatry Quarterly, 79, 249-263.

Edelson, S. M., Edelson, M. G., Kerr, D. C. R., \& Grandin, T. (1999). Behavioral and physiological effects of deep pressure on individuals with autism: A pilot study investigating the efficacy of Grandin's 'Hug Machine'. American Journal of Occupational Therapy, 53, 145-152.

Ferterl-Daly, D., Bedell, G., \& Hinojosa, J. (2001). Effects of a weighted vest on attention to task and self-stimulatory behaviors in preschoolers with pervasive developmental disorders. American Journal of Occupational Therapy, 55(6), 629-640.

Field, T., Lasko, P. M., Henteleff, T., Kabat, S., Talpins, S., \& Dowling, M. (1997). Brief report: Autistic children's attentiveness and responsivity improve after touch therapy. Journal of Autism and Developmental Disorders, 27, 333-339.

Fox, N. A., \& Polak, C. P. (2004). The role of sensory reactivity in understanding infant temperament. In R. DelCarmen-Wiggins \& A. S. Carter (Eds.), Handbook of infant, toddler, and preschool mental health assessment (pp. 105-119). NY: Oxford University Press.

Gadow, K. D., DeVincent, C. J., Pomeroy, J., \& Azizian, A. (2004). Psychiatric symptoms in preschool children with PDD and clinic and comparison samples. Journal of Autism and Developmental Disorders, 34(4), 379-393.

Garakani, A., Mathew, S. J., \& Charney, D. S. (2006). Neurobiology of anxiety disorders and implications for treatment. Mount Sinai Journal of Medicine, 73(7), 941-949.

Grillon, C. (2008). Models and mechanisms of anxiety: Evidence from startle studies. Psychopharmacology, 199, 421-437.

Juranek, J., Filipek, P. A., Berenji, G. R., Modahl, C., Osann, K., \& Spence, M. A. (2006). Association between amygdala volume and anxiety level: Magnetic resonance imaging (MRI) study in autistic children. Journal of Child Neurology, 21, 1051-1058.

Kim, J. A., Szatmari, P., Bryson, S. E., Streiner, D. L., \& Wilson, F. J. (2000). The prevalence of anxiety and mood problems among children with autism and Asperger syndrome. Autism, 4(2), 117-132.

Lainhart, J. E. (1999). Psychiatric problems in individuals with autism, their parents and siblings. International Review of Psychiatry, 11, 278-298.

Lepicard, E. M., Venault, P., Negroni, J., Perez-Diaz, F., Joubert, C., Nosten-Bertrand, M., et al. (2003). Posture and balance responses to a sensory challenge are related to anxiety in mice. Psychiatry Research, 118, 273-284.

Linderman, T. M., \& Stewart, K. B. (1999). Sensory integrative-based occupational therapy and functional outcomes in young children with pervasive developmental disorders: A single subject case study. American Journal of Occupational Therapy, 53, 207-213. 
Liss, M., Saulnier, C., Fein, D., \& Kinsbourne, M. (2006). Sensory and attention abnormalities in autistic spectrum disorders. Autism, 10(2), 155-172.

MacLeod, C., Rutherford, E., Campbell, L., Ebsworthy, G., \& Holker, L. (2002). Selective attention and emotional vulnerability: assessing the causal basis of their association through the experimental manipulation of attentional bias. Journal of Abnormal Psychology, 111, 107-123.

Markram, K., Rinaldi, T., La Mendola, D., Sandi, C., \& Markram, H. (2008). Abnormal fear conditioning and amygdala processing in an animal model of autism. Neuropsychopharmacology, 33, 901-912.

Masi, G., Millepiedi, S., Mucci, M., Poli, P., Bertini, N., \& Milantoni, L. (2004). Generalized anxiety disorder in referred children and adolescents. Journal of the American Academy of Child and Adolescent Psychiatry, 43(6), 757-760.

McIntosh, D. N., Miller, L. J., Shyu, V., \& Hagerman, R. J. (1999). Sensory modulation disruption, electrodermal responses, and functional behaviors. Developmental Medicine and Child Neurology, 41, 608-615.

Miller, L., Anzalone, M., Lane, S., Cermak, S., \& Osten, E. (2007). Concept evolution in sensory integration: a proposed nosology for diagnosis. American Journal of Occupational Therapy, 61, $135-140$.

Mobini, S., \& Grant, A. (2007). Clinical implications of attentional bias in anxiety disorders: An integrative literature review. Psychotherapy Theory, Research, Practice, Training, 44(4), 450-462.

Mogg, K., \& Bradley, B. (1998). A cognitive-motivational analysis of anxiety. Behavior Research \& Therapy, 36, 809-848.

Muris, P., Sterrneman, P., Merckelbach, H., Holdrinet, I., \& Meesters, C. (1998). Comorbid anxiety symptoms in children with pervasive developmental disorders. Journal of Anxiety Disorders, 12(4), 387-393.

Ollendick, T. H., King, N. J., \& Muris, P. (2002). Fears and phobias in children: Phenomenology, epidemiology, and aetiology. Child and Adolescent Mental Health, 7(3), 98-106.

Pfeiffer, B., \& Kinnealey, M. (2006). Treatment of sensory defensiveness in adults. Occupational Therapy International, 10(3), 175-184.

Pfeiffer, B., Kinnealey, M., Reed, C., \& Herzberg, G. (2005). Sensory modulation and affective disorders in children and adolescents with Asperger's disorder. The American Journal of Occupational Therapy, 59, 335-345.

Plappert, C. F., \& Pilz, P. K. D. (2002). Difference in anxiety and sensitization of the acoustic startle response between the two inbred mouse strains BALB/cAN and DBA/2 N. Genes, Brain, and Behavior, 1(3), 178-186.

Rauch, S. L., Shin, L. M., \& Wright, C. I. (2003). Neuroimaging studies of amygdala function in anxiety disorders. Annals of the New York Academy of Sciences, 985, 389-410.

Razran, G. (1961). The observable unconscious and the inferable conscious in current soviet psychophysiology: Interoceptive conditioning, semantic conditioning, and the orienting reflex. Psychological Review, 68(2), 81-147.

Reaven, J., \& Hepburn, S. (2003). Cognitive-behavioral treatment of obsessive-compulsive disorder in a child with Asperger syndrome. Autism, 7, 145-164.

Reynolds, S., \& Lane, S. J. (2008). Diagnostic validity of Sensory OverResponsivity: A review of the literature and case reports. Journal of Autism and Developmental Disorders, 38(3), 516-529.

Reynolds, S., Lane, S.J., \& Gennings, C. (2009). The moderating role of sensory overresponsivity in HPA activity: A pilot study with children diagnosed with ADHD. Journal of Attention Disorders. doi:10.1177/1087054708329906 (advance online publication).

Rogers, S. J., \& Ozonoff, S. (2005). What do we know about sensory dysfunction in autism? A critical review of the empirical evidence. Journal of Child Psychology and Psychiatry, 46(12), $1255-1268$.

Romancyzk, R. G., \& Gillis, J. M. (2006). Autism and physiology of stress and anxietiy. In M. G. Baron, J. Groden, G. Groden, \& L. P. Lipsitt (Eds.), Stress and coping in autism (pp. 183-204). New York: Oxford University Press.

Schoen, S. A., Miller, L. J., \& Green, K. E. (2008). Pilot study of the sensory over-responsivity scales: Assessment and inventory. American Journal of Occupational Therapy, 62, 393-406.

Silva, L. M., Schalock, M., Ayres, R., Bunse, C., \& Budden, S. (2009). Qigong massage treatment for sensory and self-regulation problems in young children with autism: A randomized controlled trial. American Journal of Occupational Therapy, 63(4), 423-432.

Sofronoff, K., Attwood, T., \& Hinton, S. (2005). A randomized controlled trail of a CBT intervention for anxiety in children with Asperger syndrome. Journal of Child Psychology and Psychiatry, 46(11), 1152-1160.

Stein, J. L., Wiedholz, L. M., Bassett, D. S., Weinberger, D. R., Zink, C. F., Mattay, V. S., et al. (2007). A validated network of effective amygdala connectivity. NeuroImage, 36, 736-745.

Sukhodolsky, D. G., Scahill, L., Gadow, K. D., Arnold, L. E., Aman, M. G., McDougle, C. J., et al. (2008). Parent-rated anxiety symptoms in children with pervasive developmental disorders: Frequency and association with core autism symptoms and cognitive functioning. Journal of Abnormal Child Psychology, 36, 117-128.

Sze, K. M., \& Wood, J. J. (2007). Cognitive behavioral treatment of comorbid anxiety disorders and social difficulties in children with high-functioning autism: A case report. Journal of Contemporary Psychotherapy, 37, 133-143.

Szeszko, P. R., MacMillan, S., McMeniman, M., Lorch, E., Madden, R., Ivey, J., et al. (2004). Amygdala volume reductions in pediatric patients with obsessive-compulsive disorder treated with paroxetine: Preliminary findings. Neuropsychopharmacology, 29, 826-832.

Weisbrot, D. M., Gadow, K. D., DeVincent, C. J., \& Pomeroy, J. (2005). The presentation of anxiety in children with pervasive developmental disorders. Journal of Child and Adolescent Psychopharmacology, 15(3), 477-496.

White, S. W., Oswald, D., Ollendick, T., \& Scahill, L. (2009). Anxiety in children and adolescents with autism spectrum disorders. Clinical Psychology Review, 29, 216-229.

Wood, J. J., Drahota, A., Sze, K., Har, K., Chiu, A., \& Langer, D. A. (2009). Cognitive behavioral therapy for anxiety in children with autism spectrum disorders: A randomized, controlled trial. Journal of Child Psychology and Psychiatry, 50(3), 224-234.

Yirmiya, N., Kasari, C., Sigman, M., \& Mundy, P. (1989). Facial expressions of affect in autistic, mentally retarded and normal children. Journal of Child Psychology and Psychiatry, 30(5), $725-735$.

Zald, D. H. (2003). The human amygdala and the emotional evaluation of sensory stimuli. Brain Research Reviews, 41, 88-123.

Zwaigenbaum, L., Bryson, S., Rogers, T., Roberts, W., Brian, J., \& Szatmari, P. (2005). Behavioral markers of autism in the first year of life. International Journal of Developmental Neurosciences, 23, 143-152. 